\title{
Side effects of Tamiflu: clues from an Asian single nucleotide polymorphism
}

\author{
Manyuan Long ${ }^{1}$ \\ ${ }^{1}$ Department of Ecology and Evolution, The University of Chicago, 1101 East 57th Street, Chicago, IL 60637, USA
}

Cell Research (2007) 17:309-310. doi: 10.1038/cr.2007.30; published online 16 April 2007

Tamiflu (Oseltamivir phosphate) seems to be a double-edged sword to some in Asia. While it is counted on against influenza and a feared avian influenza pandemic [1], the drug is also associated with side effects, ranging from neuropsychiatric, gastrointestinal, to hyperthermia and skin problems. According to a document from US Food and Drug Administration in 2005 [2], 1184 cases of side effects have been reported. Interestingly 69 out of the 75 pediatric cases were from Japan, including two teen suicides. The situation seemed to have made a gloomier turn recently. It was reported in February, 2007 that two Japanese teenagers jumped from apartment buildings after taking Tamiflu and died, bringing the total number of deaths after taking Tamiflu in Japan to $54[3,4]$. Although no direct causal relationship had been established yet, the Japan Health Ministry warned doctors about giving the drug to teenagers. In comparison, relatively few cases of severe side effects were reported from America and European countries [5]. What is wrong with this picture? It has concerned and bewildered many. The pages in this issue [6] offer one fascinating hypothesis that tries to explain the mystery using an integrated approach combining structural bioinformatic analysis and enzyme assays.

The story behind the work by Li et al. [6] exemplifies the old saying "Curiosity is the mother of invention" (and indeed a mother was involved!). In 2005 while the fear of a bird flu pandemic was looming, especially in Eastern Asia, computational biologist Liping Wei at Peking University, the senior author of this paper, was concerned for her one-year old baby son after reading the tragic news of the Japanese children who died after taking Tamiflu. Out of concern and curiosity, she decided to investigate whether and why Tamiflu might have caused the side effects and why Japan was hit the hardest. Her group focused on human sialidases which were homologues of the virus neuraminidase (NA), the intended target of the drug. Interestingly, they found that the minor allele of a nonsynonymous single nucleotide polymorphism (SNP) in human cytosolic sialidase (HsNEU2) occurs in 9.2\% of Asian and, in striking contrast, none in European and African American, according to the NCBI dbSNP database.

A hypothesis came into being that the human enzymes might also be able to bind to oseltamivir, and the identified nonsynonymous SNP may impact on the binding potential with the drug. Could this interaction with oseltamivir be the reason for the drug's side effect? The authors noticed the similarity between the reported severe side effects of oseltamivir and the known symptom of sialidase variations - several lines of evidence showed that variations of sialidase activity has significant impact on the functions and development of neurons in brain [7, 8]. The detected genetic variation of HsNEU2, if it reduces sialidase activity in the presence of oseltamivir, could be related to the severe side effects of oseltamivir.

The author's hypothesis made two testable predictions: (i) the binding ability of oseltamivir carboxylate, the active metabolite of oseltamivir, to HsNEU2 would be enhanced by the SNP and (ii) subsequently the activity of the HsNEU2 enzyme would be reduced. This hypothesis was first tested using just the published structure of HsNEU2 [9]. HsNEU2 contains the same active site as virus neuraminidase in terms of sequence and structure. The replaced residue as a result of the SNP is located nearby the active site. Molecular docking simulation showed that the amino acid change from Arg41 to Gln could increase human sialidase's binding affinity to oseltamivir carboxylate. Then, in collaboration with biochemist Xiaofeng Zheng's group at Peking University, the authors expressed both the regular and the mutant protein product of HsNEU2 in E. coli. Measuring the enzymatic activities of these HsNEU2 proteins in both the absence and 
presence of oseltamivir carboxylate, the authors confirmed that the SNP could increase human sialidase' unintended binding affinity to oseltamivir carboxylate, reducing sialidase activity. Interestingly, they also found that this SNP itself could reduce sialidase activity in the absence of oseltamivir. Thus people with this SNP likely already have dampened sialidase activity. Administration of oseltamivir might further reduce the sialidase activity in some patients to a dangerously low level.

To this point it may be tempting to jump to the conclusion that oseltamivir's interaction with the HsNEU2 SNP variant is causally related to the severe side effects reported in Japan through regulating the neuronal functions. Not so fast. The authors cautioned that their experiments were conducted in vitro and further population genetic tests with a large sample size would be required. It would also be important to genotype all four human sialidases to identify any other SNPs that may be involved in a similar mechanism. Side effects are complicated and often cannot be explained by one single factor. Nevertheless, this work offers the first testable hypothesis for the sobering and bewildering cases of reported Tamiflu side effects in Japan. The results provide a likely mechanistic cause for how the drug may impact functions of sialidases through changed structure of the enzyme in the SNP variant. Supported by structural, enzymatic, and circumstantial evidence, this study sheds first light on a mystery and points to a direction to pursue.

Patients in a high-risk population, e.g., the ones who suffered in Japan, could be tested before the drug is administered. It will be also valuable to investigate the nucleotide divergence of human sialidase genes in the Japanese population and other Asian populations and their relevancy to the side effects of Tamiflu. It may even be good news for Roche, the maker of Tamiflu, as the test may separate high-risk patients from the remaining vast majority of the population for whom the drug is generally safe and effective. The methodology adopted by this study is an integration of bioinformatics, population genetic survey, and biochemical analysis. The efficient collaboration between computational biologists and biochemists was essential.

\section{References}

1 Nelson MI, Holmes EC. The evolution of epidemic influenza. Nat Rev Genet 2007; 8:196-205.

2 Edwards ET, Truffa MM. One-year post pediatric exclusivity postmarketing adverse events review drug: Oseltamivir phosphate 2005; URL: http:/www.fda.gov/ohrms/dockets/AC/05/briefing/2005-4180b_06_01_Tamiflu\%20AE_reviewed.pdf

3 Reuters. Japan warns on teen use of Tamiflu. March 21, 2007. http://edition.cnn.com/2007/HEALTH/conditions/03/21/japan.tamiflu. reut/index.html

4 Fuyuno I. Tamiflu side effects come under scrutiny. Nature 2007; 446:358.

5 Bowles SK, Lee W, Simor AE, et al. Oseltamivir compassionate use program group. Use of oseltamivir during influenza outbreaks in Ontario nursing homes, 1999-2000. J Am Geriatr Soc 2002; 50:608-610.

$6 \mathrm{Li}$ CY, Yu Q, Ye ZQ, et al. A nonsynonymous SNP in human cytosolic sialidase in a small Asian population results in reduced enzyme activity: potential link with severe adverse reactions to oseltamivir. Cell Res 2007; 17:357-362.

7 Rodriguez JA, Piddini E, Hasegawa T, Miyagi T, Dotti CG. Plasma membrane ganglioside sialidase regulates axonal growth and regeneration in hippocampal neurons in culture. J Neurosci 2001; 21:8387-8395.

8 Becker CG, Artola A, Gerardy-Schahn R, Becker T, Welzl H, Schachner M. The polysialic acid modification of the neural cell adhesion molecule is involved in spatial learning and hippocampal long-term potentiation. J Neurosci Res 1996; 45:143-152.

9 Chavas LM, Tringali C, Fusi P, et al. Crystal structure of the human cytosolic sialidase HsNEU2. Evidence for the dynamic nature of substrate recognition. J Biol Chem 2005; 280:469-475. 